\title{
Circulating epithelial-mesenchymal transition-associated miRNAs are promising biomarkers in ovarian cancer
}

Éva Márton ${ }^{1}$, János Lukács ${ }^{2}$, András Penyige ${ }^{1,3}$, Eszter Janka ${ }^{4}$, Lídia Hegedüs ${ }^{5}$, Beáta

Soltész ${ }^{1}$, Gábor Méhes ${ }^{5}$, Róbert Póka $^{2}$, Bálint Nagy $^{1}$, Melinda Szilágyi $^{1 *}$

${ }^{1}$ Department of Human Genetics, Faculty of Medicine, University of Debrecen, Debrecen, Hungary

${ }^{2}$ Department of Obstetrics and Gynecology, Faculty of Medicine, University of Debrecen, Debrecen, Hungary

${ }^{3}$ Faculty of Pharmacology, University of Debrecen

${ }^{4}$ Department of Dermatology, Faculty of Medicine, University of Debrecen, Debrecen, Hungary

${ }^{5}$ Department of Pathology, Faculty of Medicine, University of Debrecen, Debrecen, Hungary

* Corresponding author:

Dr. Melinda Szilágyi,

University of Debrecen,

Egyetem tér 1. H-4032 Debrecen, Hungary,

Tel: +36-52-416-531,

E-mail: szlágyi.melinda@,med.unideb.hu

Running title: Circulating miRNAs in ovarian cancer

Keywords: ovarian cancer, miR200, miR34, miR203, CA125, HE4 


\begin{abstract}
Ovarian cancer is the fifth most common cause of cancer death among women that is mostly due to the difficulty of early diagnosis. Circulating miRNAs proved to be reliable biomarkers in various cancers. We screened 9 miRNAs, which are involved in epithelial-mesenchymal transition, in the plasma samples of patients with malignant $(n=28)$ or non-malignant $(n=12)$ ovarian tumors and disease-free healthy volunteers $(n=60)$ by qRT-PCR. The expression levels of miR200a, miR200b, miR200c, miR141, miR429, miR203a, miR34b (p<0.001) and miR34a $(\mathrm{p}<0.01)$ were significantly higher in the malignant samples than in healthy controls. MiR203a, miR141 ( $p<0.01)$, miR200a and miR429 ( $p<0.05)$ levels were also higher in malignant compared to non-malignant samples. ROC-AUC was the highest in the case of miR200c: 0.861 (95\%CI=0.776-0.947). Spearman's rank correlation analysis revealed positive correlation between the plasma levels of the studied miRNAs that was the highest between miR200b and miR200c $\left(r_{s}=0.774 ; p<0.001\right)$. Target analysis also suggested tight interaction between these miRNAs in the regulation of cancer development. The agreement of diagnostic tests based on miRNA levels and the standard CA125 or HE4 was weak according to Cohen's kappa values. We conclude that miR200 family members, miR34b and miR203a might be promising complementary biomarkers in ovarian cancer.
\end{abstract}




\section{Introduction}

Ovarian cancer is the fifth most common cause of cancer death among women and the most lethal form of gynecological malignancy (Siegel et al., 2018). More than 90\% of ovarian cancers have an epithelial histology and arise from cells that cover the surface of the ovary (Feeley and Wells, 2001). The 5-year survival rate ranges from 29 to $93 \%$ depending mostly on the time of diagnosis (Siegel et al., 2018). This is primarily due to the lack of early and specific symptoms. Currently, histopathology examination is considered to be the gold standard in the diagnosis of ovarian cancer. However, it has several limitations: it is difficult to obtain tumor samples, it is expensive and it is not applicable in the early diagnosis. The use of biological fluids such as blood as a source of biomarkers holds great possibilities in modern diagnostics. The non-invasive nature of liquid biopsies allows rapid diagnosis and monitoring disease progression. Moreover liquid biopsy has the potential to capture the entire genetic panorama of the tumor thus it is not influenced by the genetic heterogeneity of the tumor that is a great concern in tissue biopsies (Larrea et al., 2016).

Carbohydrate Antigen-125 (CA125) and Human Epididymis Protein 4 (HE4) are the most widely used biomarkers in ovarian cancer diagnosis (Montagnana et al., 2017). However, CA125 has low diagnostic sensitivity (50\%-62\% for early stage ovarian cancer) and limited specificity (73\%-77\%) and it is reported to be elevated in other conditions e.g. in menstruation, pregnancy and endometriosis as well (Sölétormos et al., 2016; Montagnana et al., 2017). HE4 did not contribute significantly to the improvement of ovarian cancer diagnosis either (Van Gorp et al., 2011). Circulating (or cell free) nucleic acids including miRNAs also have the potential to be useful diagnostic and prognostic biomarkers. MicroRNAs are single stranded small non-coding RNA molecules (-19-25nt in length), which play role in posttranscriptional gene regulation. They are involved in the regulation of cell cycle, apoptosis, proliferation and carcinogenesis (Hayes et al., 2014). Circulating miRNAs 
proved to be promising biomarkers in various cancers, including breast, prostate, pancreatic or colon cancer (Hayes et al., 2014; Chakraborty et al., 2016; Larrea et al., 2018). MiRNAs are frequently dysregulated in ovarian cancer and miRNA expression profiles were shown to be reliable markers in the discrimination between ovarian cancer specimens and normal ovaries (Kinose et al., 2014). However, only few publications focus on circulating miRNAs in ovarian cancer especially in Central or Eastern European populations where the incidence of ovarian cancer shows the highest rates (Reid et al., 2017).

Epithelial-mesenchymal transition (EMT) is a biological process that allows an epithelial cell to undergo multiple biochemical changes that result in a mesenchymal phenotype, which lead to enhanced migratory capacity (Kalluri and Weinberg, 2009). Ovarian carcinomas arise from epithelial cells and EMT is a crucial step in the progression to metastasis. EMT is regulated by dozens of transcription factors including members of the Snail and ZEB families. MiRNAs are also associated with the regulation of EMT among which miR200 (miR200a, miR200b, miR200c, miR141, miR429), miR34 (miR34a, miR34b, miR34c) family members and miR203 have well-established roles (Kalluri and Weinberg, 2009; Bracken et al., 2015). Our aim was to study the plasma levels of circulating miR200, miR34 family members and miR203a as biomarker candidates in epithelial ovarian cancer.

\section{Materials and methods}

\subsection{Patients}

The total of 100 blood samples (60 disease-free healthy control, 28 patients with malignant, 12 patients with non-malignant ovarian masses) were collected from the Department of Obstetrics and Gynecology, Faculty of Medicine, University of Debrecen, during 2016-2017. All ovarian cancer patients went through surgery and tissue samples were histopathologically 
diagnosed. They did not receive chemotherapy or radiotherapy prior to participation in the study. Pathological characterization of ovarian cancer was determined according to the International Federation of Gynecology and Obestrics (FIGO) criteria. All participants were informed and they signed a consent. The study protocol was approved by the Scientific and Research Ethics Committee of the Medical Research Council (ETT TUKEB) [No: 2379/2014], the research was conducted in accordance with the Declaration of Helsinki. Venous blood samples were collected in EDTA tubes and kept at $4{ }^{\circ} \mathrm{C}$ for less than 2 hours until plasma separation. Plasma was separated by two-step centrifugation $(2.500 \mathrm{~g}$ and $\left.16.000 \mathrm{~g} ; 10 \mathrm{~min}, 4^{\circ} \mathrm{C}\right)$. After separation the plasma was homogenized, aliquoted, and stored at $-80{ }^{\circ} \mathrm{C}$ until further processing.

\subsection{MiRNA extraction and quantitative real-time polymerase chain reaction ( $q R T-P C R)$}

Total RNA (including the small non-coding RNAs) was extracted from $200 \mu 1$ plasma sample by using the miRNeasy Serum/Plasma Kit (Qiagen, Hilden, Germany) according to the manufacturer's instructions. The miRNA-specific fluorometric assay using a Qubit ${ }^{\circledR} 2.0$ Fluorometer (Thermo Fischer Scientific, USA) was used to determine the concentration of extracted RNA.

The miScript PCR System (Qiagen, Hilden, Germany) was used to detect mature miRNAs. Reverse transcription of miRNAs was performed by miScript II RT Kit (Qiagen). Quantitative real-time PCR was used (Light cycler II. 1.0; Roche GmbH, Mannheim, Germany) to determine the level of miR200a-3p, miR200b-3p, miR200c-3p, miR141-3p, miR429-3p, miR34a-5p, miR34b-3p, miR34c-3p and miR203a-3p with the miScript SYBR Green PCR Kit (Qiagen). The PCR reaction mixture contained 500 pg reverse transcription products. The reaction samples were first denatured at $95^{\circ} \mathrm{C}$ for $15 \mathrm{~min}$, followed by 50 cycles of $94{ }^{\circ} \mathrm{C}$ for $15 \mathrm{sec}, 55^{\circ} \mathrm{C}$ for $30 \mathrm{sec}$ and $70^{\circ} \mathrm{C}$ for $30 \mathrm{sec}$. Finally, a melting curve was generated by taking 
fluorescent measurements every $0.2{ }^{\circ} \mathrm{C}$ for $25 \mathrm{~s}$ from $50{ }^{\circ} \mathrm{C}$ until $95^{\circ} \mathrm{C}$ to ensure the detection of a single PCR product. Cycle threshold $(\mathrm{Ct})$ values above the determinable range (up to 45) were assigned a $\mathrm{Ct}$ of 45 . All measurements were performed in triplicate and the amounts of PCR products were normalized to an internal control (miR103-3p; Kan et al., 2012). Relative expression levels were calculated by the $2^{-\Delta \mathrm{Ct}}$ method.

\subsection{Statistical analysis}

Distribution of data was analyzed by Kolmogorov-Smirnov test. Statistical significance of the expression data was determined by the Mann-Whitney U test. Spearman rank correlation was used to determine the correlation between the expression values of miRNAs $\left(r_{s}-\right.$ Spearman's rank correlation coefficient). The distribution of CA125 and HE4 values in the malignant and non-malignant samples was analyzed by Fisher's exact test. In the case of selected miRNAs sensitivity, specificity, positive predictive value (PPV), negative predictive value (NPV) and diagnostic accuracy were calculated. Receiver operating characteristics (ROC) curves and areas under the ROC curve (AUC) with corresponding upper and lower 95\% confidence intervals $(95 \% \mathrm{CI})$ were generated. In the case of some malignant samples Cohen's kappa $(\kappa)$ values were calculated to define the agreement between the diagnostic tests based on miRNAs and CA125 or HE4. Optimal cut-off points were determined by the ROC analysis based on the best balance of sensitivity and specificity. In respect of all tests the significance level was $\mathrm{p}<0.05$.

The statistical analysis was performed by SPSS 25 (SPSS package for Windows, Release 25.; SPSS, Chicago, IL, USA). The ROC-AUC graphs were generated with XLSTAT (version 2017.5, New York, NY, USA). Scatter plot was generated by GraphPad Prism 7.

\subsection{Bioinformatic analysis}


To identify experimentally validated target genes of miRNAs the miRTargetLink Human miRNA target prediction tool was used (https://ccb-web.cs.unisaarland.de/mirtargetlink/multinet.php). Target genes that showed strong interaction with the query miRNAs were established for each miRNA separately, then the overlapping network of genes targeted by two miRNAs were also generated by the tool. Functional genomic annotation was applied with functional clustering analysis on the list of the miRNA target genes using the DAVID tool with high stringency option (https://david.ncifcrf.gov; Huang et al., 2009). To rank and characterize the significance of the generated Functional Annotation Clusters the annotation term p-values and enrichment scores were used. The statistically significant enrichment of the common target genes in known biochemical/molecular pathways and their GO ontologies were also assessed by the DAVID tool.

\section{Results}

\subsection{Patient characteristics}

A total of 100 participants treated by the Department of Obstetrics and Gynecology, Faculty of Medicine, University of Debrecen were involved in this study, including 28 patients with malignant epithelial ovarian tumor, 12 patients with non-malignant ovarian masses, and 60 age-matched healthy volunteers without any ovarian disorder whose samples were used as controls in the comparisons. Disease status of all ovarian cancer patients were classified as FIGO stage I, III and IV according to imaging studies and surgical pathological findings (Table 1). CA125 and HE4 values were available for most of the malignant and nonmalignant samples (Table 1). Fisher's exact test showed statistically significant difference in the distribution of CA125 values between the malignant and non-malignant samples 
$(\mathrm{p}<0.001)$. However, the distribution did not prove to be significant in the case of HE4 values between these groups $(\mathrm{p}=0.381)$.

\subsection{Relative expression of plasma miRNAs}

In this study miR200 (miR200a, miR200b, miR200c, miR141, miR429) and miR34 (miR34a, miR34b, miR34c) family members together with miR203a were selected as candidate biomarkers in the diagnosis of ovarian cancer. MiR103 was chosen as an endogenous reference for data normalization (Kan et al. 2012).

In order to identify those miRNAs that are associated with ovarian cancer the relative expression level of miRNAs measured in the malignant and healthy samples were compared. The expression level of miR200a, miR200b, miR200c, miR141, miR429, miR34b, miR203a $(\mathrm{p}<0.001)$ and miR34a $(\mathrm{p}<0.05)$ was significantly elevated in the malignant samples (Figure 1/A and 1/B). However, the plasma level of miR34c did not show significant difference in these two groups $(\mathrm{p}=0.222$; Figure1/B).

We also studied whether these miRNAs are reliable markers in the differential diagnosis of non-malignant tumors. For this purpose the relative expression level of these miRNAs was also compared between the malignant and non-malignant samples. In this comparison the relative expression level was significantly higher in the case of miR203a, miR141 $(p<0.01)$, miR200a and miR429 $(\mathrm{p}<0.05)$ in the malignant samples (Figure1/A and 1/B). The expression level of miR200b, miR200c, miR34a and miR34b was not significantly different between the malignant and non-malignant samples $(\mathrm{p}=0.968 ; 0.2 ; 0.509 ; 0.603$ respectively; Figure 1/A and $1 / \mathrm{B})$.

\subsection{Diagnostic parameters of selected miRNAs in ovarian cancer}


To determine the diagnostic potential of selected miRNAs, sensitivity, specificity, PPV and NPV values were calculated and ROC-AUC curves were constructed based on the expression levels obtained in the malignant and healthy samples (Figure 2, Table 2). MiR34c was excluded from this analysis because it did not show significantly altered expression between these groups. ROC-AUC proved to be the largest in the case of miR200c: 0.861 (95\% $\mathrm{CI}=0.776-0.947$ ) (Figure 2, Table 2). Diagnostic accuracy was the highest in the case of miR200b (82.95\%). MiR200a, miR141 and miR429 had the highest sensitivity (85.71\%). Specificity proved to be the highest in the case of miR200b (90\%). MiR34a showed the lowest diagnostic parameters (Figure 2, Table 2).

We also constructed ROC-AUC curves using the expression data obtained from the nonmalignant and malignant samples for those miRNAs that showed significantly different expression between these groups. MiR203a could discriminate patients with malignant tumors from patients with non-malignant masses with a power AUC of 0.774 (95\% CI=0.598-0.949). ROC-AUC proved to be 0.766 (95\% CI=0.584-0.949); $0.759(95 \% \mathrm{CI}=0.556-0.962)$ and $0.720(95 \% \mathrm{CI}=0.547-0.894)$ in the case of $\mathrm{miR} 141, \mathrm{miR} 200 \mathrm{a}$ and miR429 respectively.

\subsection{Comparison of the plasma levels of miRNAs, CA125 and HE4}

Spearman rank correlation analysis was performed to assess possible correlations between the expression values of all the tested miRNAs determined in the malignant and healthy samples (Table 3/A). Positive correlation was found in all cases. Correlation proved to be the highest between miR200b and miR200c (Table 3/A). MiR200 family members showed moderate correlation with each other and with miR203a (Table 3/A). Correlation was weaker between the members of the miR200 and miR34 family (Table 3/A). It is important to mention that miR34a and miR34b showed higher correlation with each other than with miR34c (Table 
3/A). Moreover, the results of miR34c did not prove to be significant in several comparisons (Table 3/A).

We also studied the correlation of expression levels in malignant and non-malignant samples in case of those miRNAs that showed significantly different expression in these groups. Correlation followed similar tendency in this comparison as well. Positive correlation was found in all the cases. The correlation between miR141 and miR429 proved to be moderate $\left(\mathrm{r}_{\mathrm{s}}\right.$ $=0.591 ; \mathrm{p}<0.001)$. MiR141 showed slightly higher correlation with miR203a $\left(\mathrm{r}_{\mathrm{s}}=0.546\right.$; $\mathrm{p}<0.001)$ and miR200a $\left(\mathrm{r}_{\mathrm{s}}=0.555 ; \mathrm{p}<0.001\right)$ in this comparison than in the case of the malignant and healthy samples. The correlation of miR429 and miR203a also proved to be higher $\left(r_{s}=0.627 ; p<0.001\right)$ in this comparison. The correlation of miR200a was similar with $\operatorname{miR} 429\left(r_{s}=0.496 ; p<0.001\right)$ but showed lower value with miR203a in this comparison $\left(r_{s}=\right.$ $0.453 ; \mathrm{p}<0.001)$

In some malignant samples (n=21) CA125 and HE4 values were also available (Table 1).

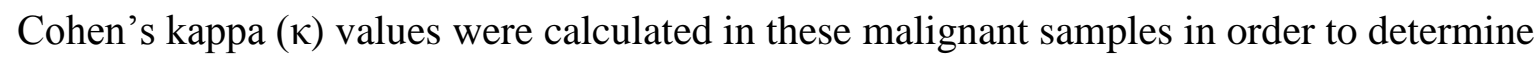
the agreement between the diagnostic tests based on selected miRNAs and the standard CA125 or HE4 markers (Table 4). The agreement was the highest between miR200b and miR200c. The agreement proved to be good between miR200c and miR34b. The agreement was moderate in several other cases between the miRNAs. HE4 showed moderate agreement with miR200b, miR200c and CA125. However, the agreement proved to be weak between the miRNAs and CA125 (Table 4).

\subsection{Target analysis of miRNAs}

In order to study the functional connections between the miRNAs by revealing their mRNA interaction partners bioinformatic tools were applied. Experimentally validated target genes shared by 2 miRNAs were analyzed by miRTargetLink. All gene lists and annotations are 
presented in the Supplementary table. Functional enrichment analysis was also performed to recognize the most significant enriched disease, GO terms and KEGG pathways associated with the miRNA target sets $(\mathrm{p}<0.05)$. In the Supplementary table only those hits are presented that are directly linked to cancer progression.

MiR200 family members are heavily interconnected through several common targets (Table 3/B). 59 target genes are shared by at least 2 members of this family (Supplementary table). Gene enrichment analysis revealed that these target genes are enriched in ovarian cancer (fold enrichment 7.2), in the regulation of endothelial cell migration (fold enrichment 26.07) and in several pathways involved in cancer (Supplementary table). Most of the targets are shared by miR200b and miR200c (Table 3/B; Supplementary table). These targets are also enriched in ovarian cancer (fold enrichment 5.39) and GO enrichment analysis revealed that the common target genes are highly enriched in the positive regulation of endothelial cell migration (fold enrichment 34.77).

MiR34 family members regulate 20 shared target genes (Supplementary table). Fold enrichment scores of these target genes proved to be higher in ovarian cancer (12.91) than that of the shared targets of miR200 family members. These genes are highly enriched in the regulation of epithelial to mesenchymal transition (fold enrichment 101.77). It is important to note that miR34a and miR34b share higher number of targets with each other than with miR34c (Table 3/B).

The interactions between the miR200 and miR34 family members or with miR203a seem to be weaker according to the lower number of shared target genes (Table 3/B, Supplementary table).

\section{Discussion}


An intriguing but poorly understood aspect of miRNAs is their presence in body fluids. Their appearance in blood shows correlation with the presence of numerous malignancies and proved to be reliable biomarkers in the diagnosis or prognosis of cancer (Hayes et al., 2014; Kinose et al., 2014). Ovarian cancer has relatively high mortality among women that is mostly due to the fact that in the majority of the patients the disease is diagnosed at advanced stage when the survival rate is only 5-21\% (Jelovac and Armstrong, 2011). Developing a minimally invasive and highly accurate diagnostic method would facilitate the diagnosis of ovarian cancer, which would also contribute to the improvement of survival chances. Here we studied the accuracy of circulating miRNAs in the diagnosis of ovarian cancer in a Caucasian (Hungarian) cohort.

We screened the expression level of 9 miRNAs in the plasma samples of patients with malignant or non-malignant ovarian tumors, and in age-matched healthy controls. Based on our results miR200 family members proved to be promising biomarkers in the diagnosis of ovarian cancer. MiR200 family members are addressed to play crucial role in the regulation of cancer initiation, angiogenesis and metastasis (Choi and $\mathrm{Ng}$, 2017). The dysregulation of miR200 family members is well-known in cancer and their circulating counterparts proved to be reliable diagnostic and prognostic markers in breast, prostate, lung, bladder or colorectal cancer (Feng et al., 2014; Liu et al., 2016; Larrea et al., 2018). Their expression is dysregulated in ovarian cancer as well and they are reported to be associated with disease stage, tumor subtypes, chemosensitivity and prognosis (Koutsaky et al., 2017). In accordance with our results circulating miR200 family members proved to be promising biomarkers in ovarian cancer in previous studies (Kan et al., 2012; Kapetanakis et al., 2015; Gao and Wu, 2015; Zuberi et al., 2015; Meng et al., 2015; Halvorsen et al.,2017; Pendelbury et al., 2017). To the best of our knowledge this is the first study that investigates circulating miR200s in a Central European population. 
According to our results high levels of circulating miR34a and miR34b were also associated with ovarian cancer among which miR34b showed better diagnostic properties. MiR34 family members are well known to regulate cell cycle, apoptosis and invasiveness in cancer (Li et al., 2014). These miRNAs proved to have direct role in the development of breast, prostate, bladder or brain cancer and miR34a is considered to be a promising therapeutic target in cancer treatment (Li et al., 2014). Elevated level of circulating miR34a and miR34b was reported in breast, lung and prostate cancer (Lodes et al., 2009; Roth et al., 2010; 2011). Their aberrant expression was also confirmed in ovarian carcinoma samples (Iorio et al., 2007; Zhang et al., 2008; Lee et al., 2009; Corney et al., 2010; Schmid et al., 2016). However, limited information is available about circulating miR34 family members in ovarian cancer. Only miR34c was under investigation previously and it was upregulated in cancer patients (Ayaz et al., 2014) - in contrast to our results.

We also detected significantly elevated expression level of miR203a in the plasma samples of patients with malignant ovarian tumor. MiR203 is a putative tumor suppressor and regulates tumor cell proliferation, invasion and angiogenesis. Its aberrant expression was confirmed in breast, colorectal, prostate cancer or in melanoma (Shao et al., 2017). Higher circulating miR203 level was reported in breast and colorectal cancer (Madhavan et al., 2016; Hur et al., 2017). Its aberrant expression was also confirmed in ovarian carcinoma samples (Iorio et al., 2007; Lee et al., 2009; Wymann et al., 2009; Wang et al., 2013; Azizmohammadi et al., 2016). However, the application of circulating miR203 as a diagnostic marker in ovarian cancer is understudied. Circulating exosomal miR203 level was found to be elevated in the sera of patients with malignant tumors compared to the sera of patients with benign disease (Taylor and Gercel-Taylor, 2008). However, miR203 level was not compared with healthy controls in this study (Taylor and Gercel-Taylor, 2008). 
One major advantage of the utilization of circulating biomarkers is that when a pelvic mass is present in patients the detection of these markers strengthens the suspicion of ovarian carcinoma. In order to identify those miRNAs that might be applicable in the differential diagnosis of non-malignant samples we also compared the expression level of miRNAs between malignant and non-malignant samples. In this comparison less miRNAs proved to be significant than when the samples of healthy volunteers were used as controls. These results suggest the followings: i) elevated miR200a, miR141, miR429 and miR203a levels might be reliable biomarkers in the discrimination of non-malignant masses from malignant tumors; ii) non-malignant samples might not be applicable as controls because comparison of malignant samples to non-malignants might produce different results than using healthy samples as controls. However, further studies are needed to strengthen this hypothesis with larger samples size.

We also studied the agreement between the diagnostic tests based on miRNAs and the standard biomarkers CA125 and HE4. Although the agreement of some miRNAs was moderate with HE4 they showed weak agreement with CA125. However, higher agreement was observed between the diagnostic tests based on miRNAs. The Cohen's kappa coefficient was the highest between the diagnostic tests based on miR200b and miR200c. We have to note that this analysis was performed only in the case of malignant samples. Previous publications that studied the correlation between miRNAs and CA125 showed controversial results. According to some publications circulating miRNA levels are not consistent with CA125 (Resnick et al., 2009; Kapetanakis et al., 2015) but others suggest positive correlation (Meng et al., 2015; 2016). This discrepancy might be explained by the fact that the diagnostic properties of CA125 are far from perfect (Montagnana et al., 2017) that is also true for single miRNAs. It is important to note that multivariate diagnostic tests that combine circulating miRNAs with CA125 or HE4 had higher efficiency than single tests in several studies (Elias 
et al., 2017; Montagnana et al., 2017; Ren et al., 2018, Shah et al., 2018). We conclude that miR200 family members, miR203a and miR34b are promising candidates for multivariate diagnostic tests based on the combination of miRNAs with each other or with CA125 and HE4 markers. Further studies are needed with higher sample size in order to strengthen this hypothesis.

We also studied the regulatory relationship of these miRNAs through assessing their shared target genes and by calculating the correlation between their expression levels. We found high agreement between the correlation of their plasma level and the number of target genes they share. Strong positive correlation was identified between the members of the miR200 family, among which miR200b and miR200c expression levels showed the highest correlation coefficient. Target analysis revealed that these miRNAs co-regulate several genes involved in cancer progression. Similar phenomenon was observed in the case of miR34a and miR34b. It is also important to note that weaker correlation was found between those miRNAs that shared less targets e.g. between miR34 and miR200 family members or between miR34b and miR34c. Our results suggest that the expression and extracellular trafficking of those miRNAs that are tightly connected through shared targets in miRNA-target gene interaction networks might be co-regulated. This regulation might be facilitated by the formation of exosomes that proved to be important in the delivery of these miRNAs according to previous results (Taylor and Gercel-Taylor, 2008). It is also important to note that the release of miRNAs - partly through the formation of exosomes - seems to be under active regulation (Collino et al., 2010). Our further aim is to shed light on the molecular details of this regulation that might lead to the development of more efficient diagnostic tests in ovarian cancer.

\section{Conflict of interest}


The authors declare that they have no known competing financial interests or personal relationships that could have appeared to influence the work reported in this paper.

\section{Author contribution}

MS formulated the main ideas in the paper with BN. MÉ made the laboratory work including miRNA isolation and quantification. EJ was responsible for the statistical analysis and AP helped with the bioinformatic tools. JL, RP and BS were responsible for plasma sample collection. LH and GM made the pathological classification of the samples. MS wrote the paper with the help of BN.

\section{Acknowledgment}

The authors are grateful to Diana Maricela Herrera Villarroel, Réka Szabó, Edina Szilágyi and KatalinTrefán for their excellent laboratory assistance. This work was supported by the Hungarian NTP-NFTÖ-18-B-0377 scholarship program.

\section{Compliance with Ethical Standards}

Research involving Human Participants and/or Animals: ethical permission was obtained from the Ministry of Health (TUKEB). Informed consent: was obtained from each participant

\section{References}

Ayaz, L., Çayan, F., Balci, Ş., Görür, A., Akbayir, S., YıldırımYaroğlu, H., DoğruerUnal, N., Tamer, L., 2014.Circulating microRNA expression profiles in ovarian cancer. J

ObstetGynaecol. 34, 620-624. 
Azizmohammadi, S., Azizmohammadi, S., Safari, A., Kosari, N., Kaghazian, M., Yahaghi, E., Seifoleslami, M., 2016. The role and expression of miR-100 and miR-203 profileasprognosticmarkersinepithelialovariancancer. Am J Transl Res. 8, 2403-2410.

Bracken, C.P.,Khew-Goodall, Y., Goodall, G.J., 2015. Network-Based Approaches to Understand the Roles of miR-200 and Other microRNAs in Cancer. Cancer Res 75, 25942599.

Chakraborty, C., Das, S., 2016. Profiling cell-free and circulating miRNA: a clinical diagnostic tool for different cancers. Tumor Biol. 37, 5705-5714.

Choi, P.W.,Ng, S.W., 2017. The Functions of MicroRNA-200 FamilyinOvarianCancer: BeyondEpithelial-MesenchymalTransition. Int. J. Mol. Sci. 18(6), pii: E1207

Collino, F., Deregibus, M.C., Bruno, S., Sterpone,L., Aghemo, G., Viltono, L., Tetta, C., Camussi, G., 2010. Microvesicles derived from adult human bone marrow and tissue specific mesenchymal stem cells shuttle selected pattern of miRNAs. PLoS One.5, e11803.

Corney, D.C., Hwang, C.I., Matoso, A., Vogt, M., Flesken-Nikitin, A., Godwin, A.K., Kamat, A.A., Sood, A.K., Ellenson, L.H., Hermeking, H., Nikitin, A.Y., 2010.Frequent downregulation of miR-34 family in human ovarian cancers. Clin Cancer Res. 16, 1119-1128.

Elias, K.M., Fendler, W., Stawiski, K., Fiascone, S.J., Vitonis, A.F., Berkowitz, R.S., Frendl, G., Konstantinopoulos, P., Crum, C.P., Kedzierska, M., Cramer, D.W., Chowdhury, D., 2017. Diagnostic potential for a serum miRNA neural network for detection of ovarian cancer. Elife. 6, e28932.

Feeley, K.M., Wells, M., 2001. Precursor lesions of ovarian epithelial malignancy. Histopathology 38, 87-95. 
Feng, X., Wang, Z., Fillmore, R., Xi, Y., 2014. MiR-200, a new star miRNA in human cancer. Cancer Lett. 344, 166-173.

Gao, Y.C., Wu, J., 2015. MicroRNA-200c and microRNA 141 as potential diagnostic and prognostic biomarkers for ovarian cancer. Tumor Biol. 36, 4843-4850.

Halvorsen, A.R., Kristensen, G., Embleton, A., Adusei, C., Barretina-Ginesta, M.P., Beale, P., Helland, Å., 2017. Evaluation of Prognostic and Predictive Significance of Circulating MicroRNAs in Ovarian Cancer Patients. Dis Markers. 2017, 3098542.

Hayes, J,, Peruzzi, P.P., Lawler, S., 2014. MicroRNAs in cancer: biomarkers, functions and therapy. Trends Mol Med. 20, 460-469.

Huang, D.W., Sherman, B.T., Lempicki, R.A ., 2009. Bioinformatics enrichment tools: paths toward the comprehensive functional analysis of large gene lists. Nucleic Acids Res 37, 1-13.

Hur, K., Toiyama, Y., Okugawa, Y., Ide, S., Imaoka, H., Boland, C.R., Goel, A., 2015. Circulating microrna-203 predicts prognosis and metastasis in human colorectal cancer. Gut $66,654-665$.

Iorio, M.V., Visone, R., Di Leva, G., Donati, V., Petrocca, F., Casalini, P., Taccioli, C., Volinia, S., Liu, C.G., Alder, H., Calin, G.A., Ménard, S., Croce, C.M., 2007. MicroRNA signatures in human ovarian cancer. Cancer Res. 67(18), 8699-707.

Jelovac, D., Armstrong, D.K ., 2011. Recent progress in the diagnosis and treatment of ovarian cancer. CA. Cancer J Clin 61, 183-203.

Kan, C.W., Hahn, M.A., Gard, G.B., Maidens, J., Huh, J.Y., Marsh, D.J., Howell, V.M., 2012. Elevated levels of circulating microRNA-200 family members correlate with serous epithelial ovarian cancer. BMC Cancer. 12, 627. 
Kalluri, R., Weinberg, R.A., 2009.The basics of epithelial-mesenchymaltransition. J ClinInvest. 119(6), 1420-8.

Kapetanakis, N.I., Uzan, C., Jimenez-Pailhes, A.S., Gouy, S., Bentivegna, E., Morice, P., Caron, O., Gourzones-Dmitriev, C., Le Teuff, G., Busson, P., 2015. Plasma miR-200b in ovarian carcinoma patients: distinct pattern of pre/post-treatment variation compared to CA125 and potential for prediction of progression-free survival. Oncotarget 6(34), 36815-24.

Kinose, Y., Sawada, K., Nakamura, K., Kimura, T., 2014. The role of microRNAs in ovarian cancer. Biomed Res Int. 2014, 249393.

Koutsaki, M., Libra, M., Spandidos, D.A., Zaravinos, A., 2017. The miR-200 family in ovarian cancer. Oncotarget. 8(39), 66629-66640.

Larrea, E., Sole, C., Manterola, L., Goicoechea, I., Armesto, M., Arestin, M., Caffarel, M.M., Araujo, A.M., Araiz, M., Fernandez-Mercado, M., Lawrie, C.H., 2016. New ConceptsinCancerBiomarkers: Circulating miRNAs inLiquidBiopsies. Int. J. Mol. Sci. 17(5), pii: E627.

Lee, C.H., Subramanian, S., Beck, A.H., Espinosa, I., Senz ,J,. Zhu, S,X., Huntsman, D., van de Rijn, M., Gilks, C.B., 2009. MicroRNA profiling of BRCA1/2 mutation-carrying and nonmutation-carryinghigh-gradeserouscarcinomas of ovary. PLoSOne. 4(10), e7314.

Li, X.J., Ren, Z.J., Tang, J.H., 2014. MicroRNA-34a: a potential therapeutic target in human cancer. Cell Death Dis. 5, e1327.

Liu, X., Zhang, J., Xie, B., Li, H., Shen, J., Chen, J., 2016. MicroRNA-200 Family Profile: A Promising Ancillary Tool for Accurate Cancer Diagnosis. Am J Ther. 23(2), e388-97. 
Lodes, M.J., Caraballo, M., Suciu, D., Munro, S., Kumar, A., Anderson, B., 2009. Detection of cancer with serum miRNAs on an oligonucleotide microarray. PLoS ONE, 4, e6229.

Madhavan, D., Peng, C., Wallwiener, M., Zucknick, M., Nees, J., Schott, S., Rudolph, A., Riethdorf, S., Trumpp, A., Pantel, K., Sohn, C., Chang-Claude, J., Schneeweiss, A.,

Burwinkel, B., 2016. Circulating mirnas with prognostic value in metastatic breast cancer and for early detection of metastasis. Carcinogenesis 37, 461-470.

Meng, X., Joosse, S.A., Müller, V., Trillsch, F., Milde-Langosch, K., Mahner, S., Geffken, M., Pantel, K., Schwarzenbach, H., 2015. Diagnostic and prognostic potential of serum miR7, miR-16, miR-25, miR-93, miR-182, miR-376a and miR-429 in ovarian cancer patients. Br. J. Cancer. 113(9), 1358-66.

Meng, X., Müller, V., Milde-Langosch, K., Trillsch, F., Pantel, K., Schwarzenbach, H., 2016. Diagnostic and prognostic relevance of circulating exosomal miR-373, miR-200a, miR-200b and miR-200c in patients with epithelial ovarian cancer. Oncotarget.7, 16923-35.

Montagnana, M., Benati, M., Danese, E., 2017.Circulating biomarkers in epithelial ovarian cancer diagnosis: from present to future perspective. Ann. Transl. Med. 5(13), 276.

Pendlebury, A., Hannan, N.J., Binder, N., Beard, S., Mcgauran, M., Grant, P., Tong, S., Whitehead, C.L., 2017. The circulating microRNA-200 family in whole blood are potential biomarkers for high-grade serous epithelial ovarian cancer. Biomed Rep. 6, 319-322.

Reid, B.M., Permuth, J.B., Sellers, T.A., 2017.Epidemiology of ovariancancer: a review. Cancer Biol Med. 14(1), 9-32. 
Ren, X., Zhang, H., Cong, H., Wang, X., Ni, H., Shen, X., Ju, S., 2018. Diagnostic Model of Serum miR-193a-5p, HE4 and CA125 Improves the Diagnostic Efficacy of Epithelium Ovarian Cancer. Pathol Oncol Res. 24(4), 739-744.

Resnick, K.E., Alder, H., Hagan, J.P., Richardson, D.L., Croce, C.M., Cohn, D.E., 2009. The detection of differentially expressed microRNAs from the serum of ovarian cancer patients using a novel real-time PCR platform. Gynecol Oncol. 112, 55-59.

Roth, C., Rack, B., Muller, V., Janni,W., Pantel, K., Schwarzenbach, H., 2010. Circulating microRNAs as blood-based markers for patients with primary and metastatic breast cancer.Breast Cancer Res. 12(6), R90.

Roth, C., Kasimir-Bauer, S., Pantel, K., Schwarzenbach, H., 2011. Screening for circulating nucleic acids and caspase activity in the peripheral blood as potential diagnostic tools in lung cancer. Mol. Oncol. 5, 281-291.

Shah, J.S., Gard, G.B., Yang, J., Maidens, J., Valmadre, S., Soon, P.S., Marsh, D.J., 2018. Combining serum microRNA and CA-125 as prognostic indicators of preoperative surgical outcome in women with high-grade serous ovarian cancer. Gynecol Oncol. 148(1), 181-188.

Shao, Y., Gu, W.,Ning, Z., Song, X., Pei, H., Jiang, J., 2017. Evaluating the Prognostic Value of microRNA-203 in Solid Tumors Based on a Meta-Analysis and the Cancer Genome Atlas (TCGA) Datasets. Cell Physiol Biochem. 41(4), 1468-1480.

Schmid, G., Notaro, S., Reimer, D., Abdel-Azim, S., Duggan-Peer, M., Holly, J., Fiegl, H; Rössler, J., Wiedemair, A., Concin, N., Altevogt, P., Marth, C., Zeimet, A.G., 2016.

Expression and promoter hypermethylation of miR-34a in the various histological subtypes of ovarian cancer. BMC Cancer, 16, 102. 
Siegel, R.L., Miller, K.D., Jemal, A., 2018. Cancer statistics, CA. Cancer J Clin. 68, 7-30.

Sölétormos, G., Duffy, M.J., Hassan, S.O.A., Verheijen, R.H.M., Tholander, B., Bast, R.C. Jr., Gaarenstroom, K.N., Sturgeon, C.M., Bonfrer, J.M., Petersen, P.H., Troonen, H., CarloTorre, G., Kantyh Kulpa, J., Tuxen, M.K., Molina, R., 2016. Clinical use of cancer biomarkers in epithelial ovarian cancer: updated guidelines from the European Group on Tumor Markers. Int J Gynecol Cancer, 26, 43-51.

Taylor, D.D., Gercel-Taylor, C., 2008. MicroRNA signatures of tumor-derived exosomes as diagnostic biomarkers of ovarian cancer. Gynecol. Oncol. 110, 13-21.

Van Gorp, T., Cadron, I., Despierre, E., Daemen, A., Leunen, K., Amant, F., Timmerman, D., De Moor, B., Vergote, I., 2011. HE4 and CA125 as a diagnostic test in varian cancer: prospective validation of the Risk of Ovarian Malignancy Algorithm. Br J Cancer. 104, 86370.

Wang, S., Zhao, X., Wang, J., Wen, Y., Zhang, L., Wang, D., Chen, H., Chen, Q., Xiang, W., 2013.Upregulation of microrna-203 is associated with advanced tumor progression and poor prognosis in epithelial ovarian cancer. Med. Oncol. 30(3), 681.

Wyman, S.K., Parkin, R.K., Mitchell, P.S., Fritz, B.R., O'Briant, K., Godwin, A.K., Urban, N., Drescher, C.W., Knudsen, B.S., Tewari, M., 2009. Repertoire of microRNAs in epithelial ovarian cancer as determined by next generation sequencing of small RNA cDNA libraries. PLoSOne 4(4), e5311.

Zhang, L., Volinia, S., Bonome, T., Calin, G.A., Greshock, J., Yang, N., Liu, C.G., Giannakakis, A., Alexiou, P., Hasegawa, K., Johnstone, C.N., Megraw, M.S., Adams, S., Lassus, H., Huang, J., Kaur, S., Liang, S., Sethupathy, P., Leminen, A., Simossis, V.A., Sandaltzopoulos, R., Naomoto, Y., Katsaros, D., Gimotty, P.A., DeMichele, A., Huang, Q., 
Bützow, R., Rustgi, A.K., Weber, B.L., Birrer, M.J., Hatzigeorgiou, A.G., Croce, C.M., Coukos, G., 2008. Genomic and epigenetic alterations deregulate microRNA expression in human epithelial ovarian cancer. ProcNatlAcadSci U S A. 105(19), 7004-9.

Zuberi, M., Mir, R., Das, J., Ahmad, I., Javid, J.,Yadav, P., Masroor, M., Ahmad, S., Ray, P.C., Saxena, A., 2015. Expression of serum miR-200a, miR-200b, and miR-200c as candidate biomarkers in epithelial ovarian cancer and their association with clinicopathologicalfeatures. Clin Transl Oncol 17, 779-87. 


\section{Figure legends}

Figure 1 Plasma level of miRNAs.

A) Scatter plot of miR200 family members for patients with malignant tumor (M), nonmalignant masses (NM) and in healthy controls $(\mathrm{H})$.

B) Scatter plot of miR34 family members and miR203a for patients with malignant tumor (M), non-malignant masses (NM) and in healthy controls $(\mathrm{H})$.

Figure 2 ROC curves of miRNAs.

\section{Supplementary table}

Annotation of experimentally validated target genes that are shared by at least 2 members of the miR200 family (page 1), by miR200b and miR200c (page 2), by at least 2 members of the miR34 family (page 3), by at least 1 member of the miR200 family with at least 1 member of the miR34 family (page 4), by at least 1 member of the miR200 family with miR203a (page 5), or by at least 1 member of the miR34 family with miR203a (page 6). Enrichment analysis is also presented including the most significantly enriched disease, GO terms and KEGG pathways associated with the miRNA target sets $(\mathrm{p}<0.05)$. 
Table 1 Clinical characteristics of patients with malignant ovarian tumor, non-malignant ovarian masses and healthy volunteers in the study.

\begin{tabular}{|c|c|c|c|c|}
\hline \multicolumn{2}{|l|}{ Parameter } & Malignant & Non-malignant & Healthy control \\
\hline \multicolumn{2}{|l|}{ Age (years) } & $57.03 \pm 9.56$ & $57.27 \pm 11.76$ & $56.17 \pm 12.18$ \\
\hline \multicolumn{5}{|l|}{ Histological type } \\
\hline \multicolumn{2}{|l|}{$\begin{array}{l}\text { Cystadenocarcinoma } \\
\text { papillare serosum }\end{array}$} & 21 & & \\
\hline \multicolumn{2}{|l|}{$\begin{array}{l}\text { Cystadenocarcinoma } \\
\text { serosum }\end{array}$} & 3 & & \\
\hline \multicolumn{2}{|l|}{$\begin{array}{l}\text { Adenocarcinoma } \\
\text { papillare serosum }\end{array}$} & 2 & & \\
\hline \multicolumn{2}{|l|}{ Cysta serosa simplex } & & 3 & \\
\hline \multicolumn{2}{|l|}{$\begin{array}{l}\text { Cystadenoma } \\
\text { serosum }\end{array}$} & & 2 & \\
\hline \multicolumn{2}{|l|}{$\begin{array}{l}\text { Cystadenoma } \\
\text { mucinosum }\end{array}$} & & 1 & \\
\hline \multicolumn{2}{|l|}{$\begin{array}{l}\text { Cystadenofibroma } \\
\text { serosum }\end{array}$} & & 2 & \\
\hline \multicolumn{2}{|l|}{ Fibroma ovarii } & & 1 & \\
\hline \multicolumn{2}{|l|}{ Ovarium fibrosis } & & 1 & \\
\hline \multicolumn{2}{|l|}{$\begin{array}{l}\text { Salpingitis } \\
\text { fibrosa }\end{array}$} & & 1 & \\
\hline \multicolumn{2}{|l|}{ Cysta follicularis } & & 1 & \\
\hline \multirow[t]{8}{*}{ FIGO stage } & I. & 3 & & \\
\hline & $\mathrm{a}$ & 2 & & \\
\hline & $\mathrm{c}$ & 1 & & \\
\hline & III. & 18 & & \\
\hline & $\mathrm{a}$ & 2 & & \\
\hline & $\mathrm{b}$ & 3 & & \\
\hline & $\mathrm{c}$ & 13 & & \\
\hline & IV. & 7 & & \\
\hline
\end{tabular}




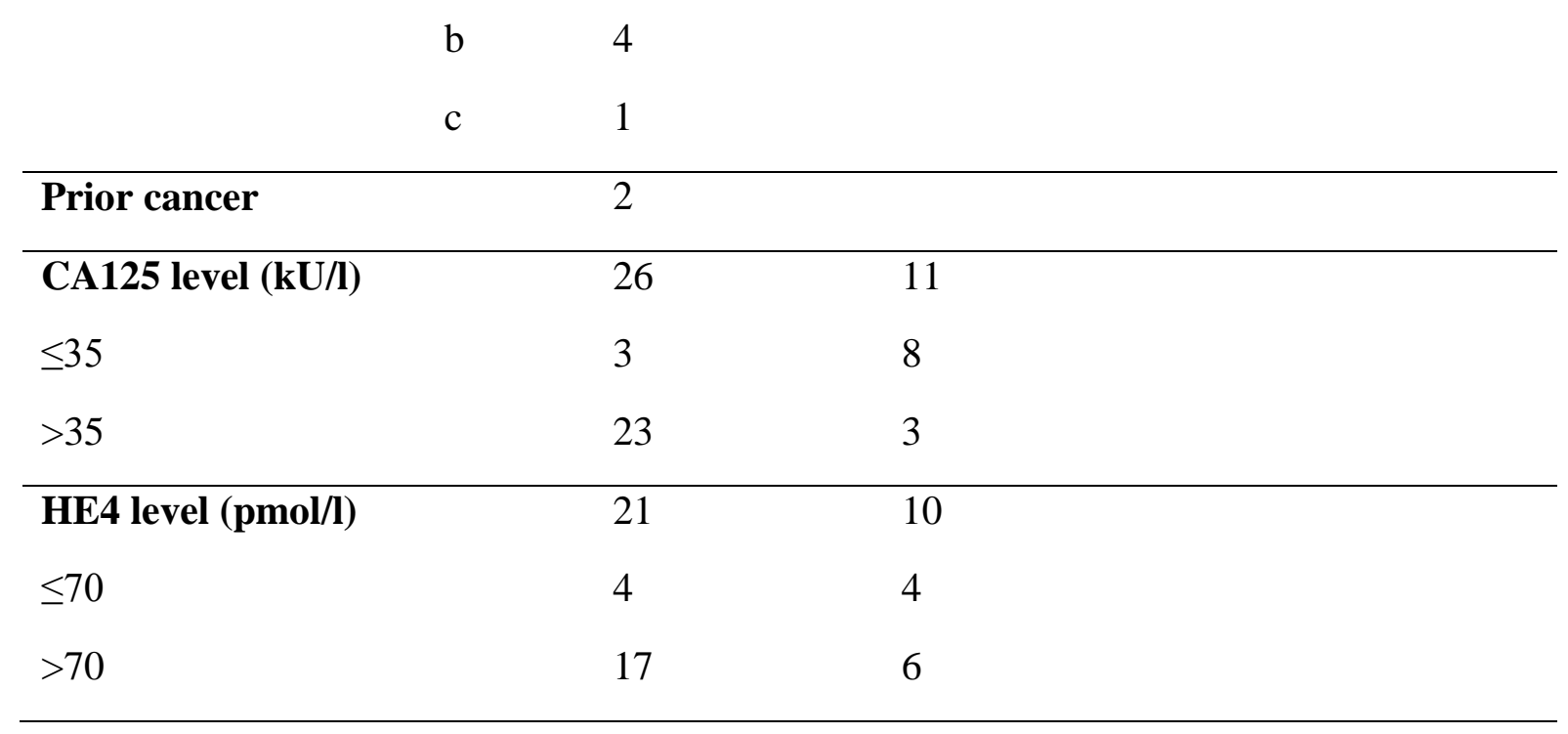


Table 2 Diagnostic parameters of miRNAs

\begin{tabular}{lllllll}
\hline & AUC & $\begin{array}{l}\text { Diagnostic } \\
\text { accuracy }\end{array}$ & Sensitivity & Specificity & PPV & NPV \\
\hline miR200a & 0.845 & $84.09 \%$ & $71.43 \%$ & $90 \%$ & $76.92 \%$ & $87.1 \%$ \\
miR200b & 0.822 & $82.95 \%$ & $67.86 \%$ & $90 \%$ & $76 \%$ & $85.71 \%$ \\
miR200c & 0.861 & $81.82 \%$ & $71.43 \%$ & $86.67 \%$ & $71.43 \%$ & $86.67 \%$ \\
miR141 & 0.825 & $81.82 \%$ & $85.71 \%$ & $80 \%$ & $66.67 \%$ & $92.31 \%$ \\
miR429 & 0.791 & $77.27 \%$ & $64.29 \%$ & $83.33 \%$ & $64.29 \%$ & $83.33 \%$ \\
miR203a & 0.831 & $84.09 \%$ & $82.14 \%$ & $85 \%$ & $71.88 \%$ & $91.07 \%$ \\
miR34a & 0.655 & $60.23 \%$ & $57.14 \%$ & $61.67 \%$ & $41.03 \%$ & $75.51 \%$ \\
miR34b & 0.832 & $73.86 \%$ & $71.43 \%$ & $75 \%$ & $57.14 \%$ & $84.91 \%$ \\
\hline
\end{tabular}


Table 3 Interactions between miRNAs.

2 A: Spearman's rank correlation coefficients $\left(r_{s}\right)$ between the expression levels of miRNAs. \# $p>0.05$. In all the other cases $p<0.05$

B: Number of validated target genes shared by miRNAs. The analysis was performed by miRTargetLink.

4

\begin{tabular}{lccccccccc}
\hline miR200a & - & 0.609 & 0.590 & 0.463 & 0.436 & 0.541 & 0.324 & 0.325 & $0.099 \#$ \\
miR200b & 25 & - & 0.774 & 0.554 & 0.423 & 0.650 & 0.474 & 0.284 & 0.290 \\
miR200c & 23 & 43 & - & 0.497 & 0.453 & 0.673 & 0.378 & 0.363 & $0.209 \#$ \\
miR141 & 23 & 19 & 19 & - & 0.616 & 0.471 & 0.399 & 0.415 & $0.192 \#$ \\
miR429 & 22 & 24 & 21 & 18 & - & 0.446 & 0.470 & 0.351 & 0.264 \\
miR203a & 4 & 5 & 5 & 3 & 1 & - & 0.315 & 0.241 & $0.162 \#$ \\
miR34a & 3 & 7 & 6 & 2 & 4 & 7 & - & 0.384 & 0.276 \\
miR34b & 1 & 4 & 3 & 0 & 3 & 4 & 19 & - & $0.018 \#$ \\
miR34c & 1 & 1 & 0 & 0 & 0 & 0 & 2 & 0 & - \\
\hline
\end{tabular}

5 
8 Table 4 Cohen's kappa values $(\kappa)$ between the pairwise comparison of the agreement between the diagnostic tests based on miRNAs and CA125 9 or HE4.

\begin{tabular}{lccccccccccc}
\hline & miR200a & miR200b & miR200c & miR141 & miR429 & miR203a & miR34a & miR34b & CA125 & HE4 \\
\hline miR200a & - & 0.529 & 0.375 & 0.173 & 0.475 & 0.391 & 0.316 & 0.077 & 0.385 & $<0.01$ \\
miR200b & 0.529 & - & 0.886 & 0.087 & 0.538 & 0.5 & 0.516 & 0.571 & $<0.01$ & 0.39 \\
miR200c & 0.375 & 0.886 & - & 0.125 & 0.386 & 0.314 & 0.417 & 0.667 & 0.028 & 0.474 \\
miR141 & 0.173 & 0.087 & 0.125 & - & 0.504 & 0.323 & $<0.01$ & 0.087 & 0.323 & $<0.01$ \\
miR429 & 0.475 & 0.538 & 0.386 & 0.504 & - & 0.696 & 0.121 & 0.308 & 0.087 & 0.013 \\
miR203a & 0.391 & 0.5 & 0.314 & 0.323 & 0.696 & - & 0.31 & 0.25 & $<0.01$ & $<0.01$ \\
miR34a & 0.316 & 0.516 & 0.417 & $<0.01$ & 0.121 & 0.31 & - & 0.516 & $<0.01$ & 0.019 \\
miR34b & 0.077 & 0.571 & 0.667 & 0.087 & 0.308 & 0.25 & 0.516 & - & $<0.01$ & 0.16 \\
CA125 & 0.385 & $<0.01$ & 0.39 & 0.323 & 0.087 & $<0.01$ & $<0.01$ & $<0.01$ & - & 0.483 \\
HE4 & $<0.01$ & 0.39 & 0.474 & $<0.01$ & 0.013 & $<0.01$ & 0.019 & 0.16 & 0.483 & - \\
\hline
\end{tabular}


Figure 1
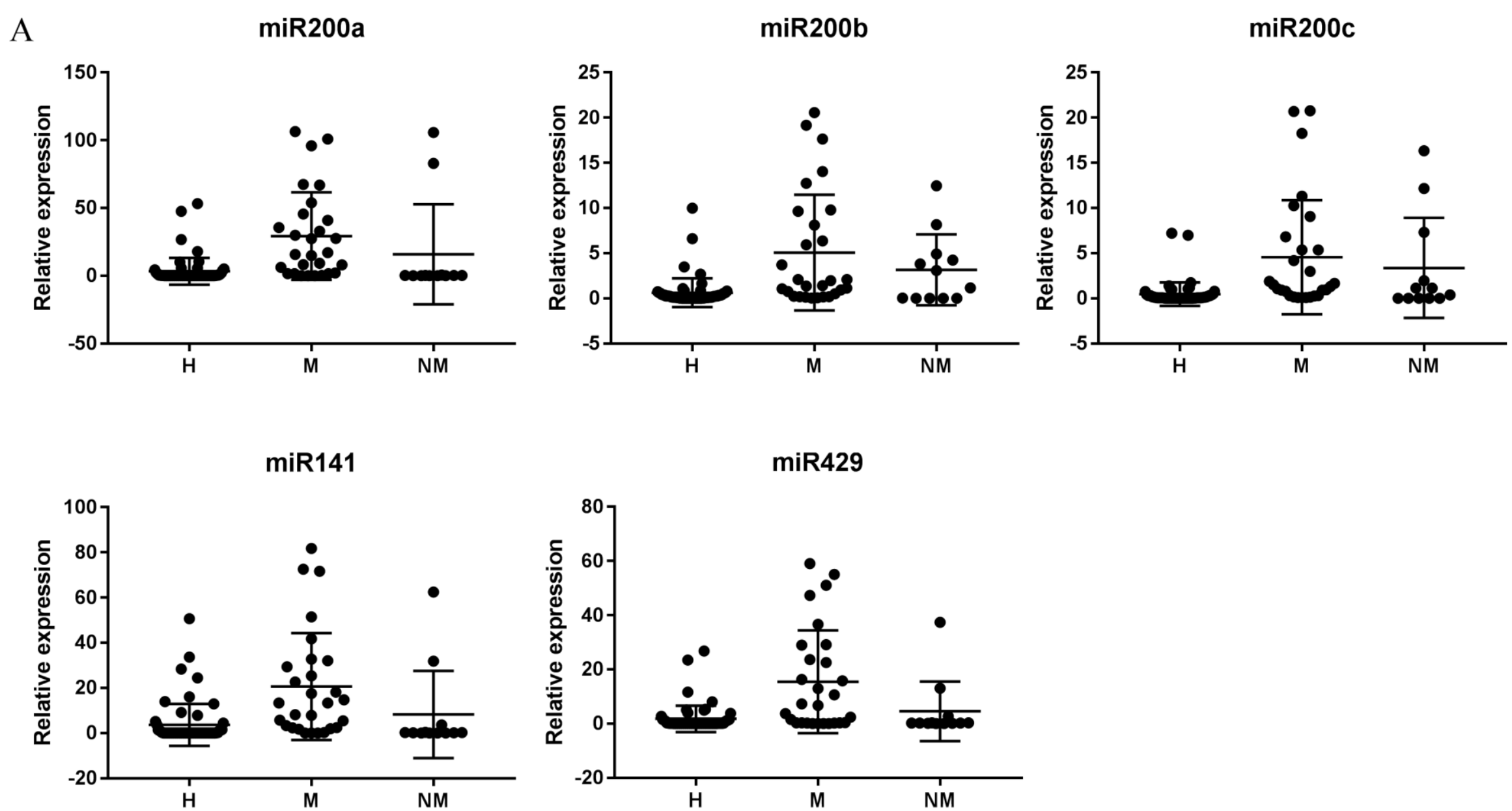

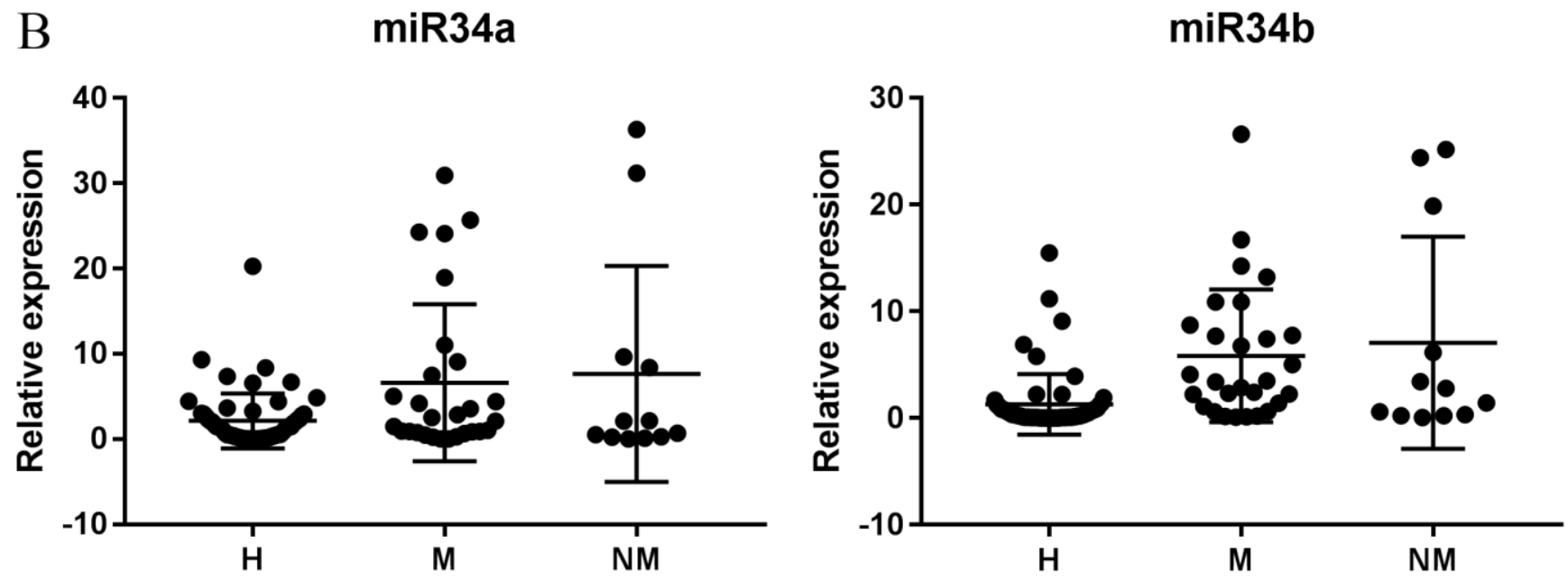

miR34c

miR203a
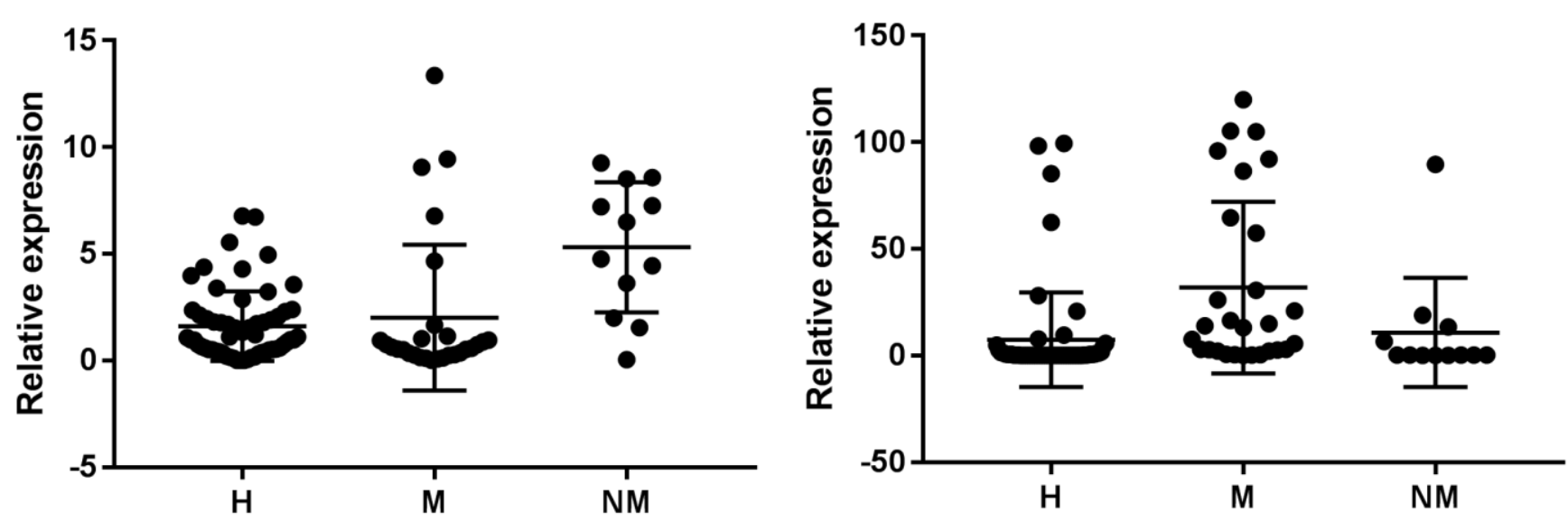
Figure 2
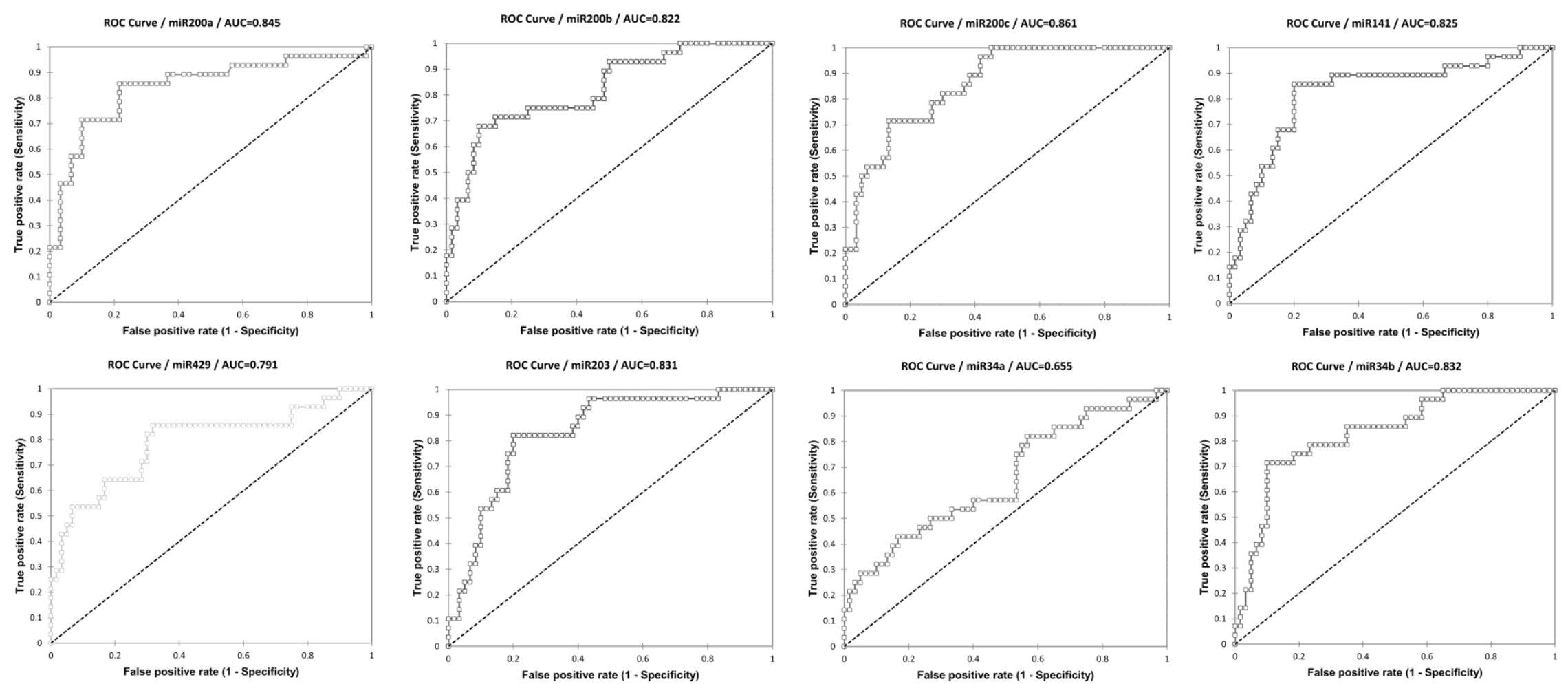